\title{
El marco jurídico, mediático y político de la conciliación en España. Desde 1999 hasta 2009¹
}

\author{
Cristina GuIRAO MiróN \\ Universidad de Murcia \\ cguirao@um.es \\ Marta MARTín LLAGUNO \\ Universidad de Alicante \\ marta.martin@ua.es
}

Recibido: $31 / 07 / 2012$

Aceptado: 24/10/2012

\section{Resumen}

Este estudio tiene como objetivo describir cronológicamente el marco jurídico, mediático y político que se ha construido en España entorno al conflicto familiar y laboral. Para ello, se ha partido de un análisis de las principales leyes que afectan a la conciliación de la vida laboral y familiar, ley de Conciliación y ley de Igualdad; de las iniciativas parlamentarias, extraídas de las actas del diario de sesiones del Congreso de los Diputados desde marzo de 1996 a marzo de 2008 (VI, VII y VIII legislatura), y de cómo los medios de comunicación, la prensa, ha enfocado el problema de la conciliación, cuáles han sido los encuadres fundamentales. El objetivo ha sido identificar si ha habido algún sesgo de género y, en último termino, definir cómo se ha construido el problema de la conciliación en España.

Palabras clave: conciliación vida laboral y familiar, marco legal, encuadres, iniciativas parlamentarias, género.

The legal, media and political of the conflict between work and family. Review of the construction work-life balance in Spain from 1999 to 2009

\begin{abstract}
This study aims to describe chronologically the legal, political and media that has been built in Spain around the work and family conflict. To do this, we have started from an analysis of the major laws affecting the reconciliation of work and family life; parliamentary initiatives on this subject have happened over the last three legislatures and how the media, the press, has approached the problem of reconciliation, what have been the key frames. The aim was to identify whether there was any gender bias and, ultimately define how built the problem of reconciliation in Spain.
\end{abstract}

Keywords: work life balance, legal framework, framing, parliamentary initiatives, gender

\section{Referencia normalizada}

GUIRAO MIRÓN, Cristina y MARTÍN LLAGUNO, Marta (2012): "El marco jurídico, mediático y político de la conciliación en España. Desde 1999 hasta 2009”. Estudios sobre el mensaje periodístico. Vol. 18, núm. especial octubre, págs.: 475-485. Madrid, Servicio de Publicaciones de la Universidad Complutense.

Sumario: 1. Introducción. 2. Metodología. 3. Fuentes. 4. Desarrollo; 4.1. Respecto al análisis jurídico; 4.1.1. La Ley 39/1999 de Conciliación Vida familiar y Laboral; 4.1.2. Ley 3/2007 para la Igualdad Efectiva de Mujeres y Hombres; 4.2. Respecto al análisis mediático; 4.3. Respecto al análisis político. 5. Conclusiones. 6. Referencias bibliográficas. 7. Legislación.

${ }^{1}$ Este artículo forma parte de los resultados del proyecto I+D+I, "La construcción social del problema del conflicto trabajo-familia. Análisis mediático, político y de la realidad" financiado por el Instituto de la Mujer en la convocatoria 2004-2007 dirigido por Marta Martín Llaguno y disponible en el Instituto de la Mujer (memoria 865). 


\section{Introducción}

Entre 1999 y 2009 España construye un marco legal, político y mediático que traduce una visibilización creciente del conflicto de la conciliación y que se concreta en la implantación de políticas públicas para paliar este conflicto. Ya desde la conferencia de Beijing, punto de partida de las estrategias de mainstreaming de género, se detectó una creciente preocupación mundial por el problema de la conciliación vida y trabajo. En Europa son numerosas la directivas que encaraban este conflicto y recomendaban a los países miembros de la unión incorporar medidas de conciliación a sus respectivas legislaciones.

Sucede que en sí mismo, el concepto es entendido de múltiples maneras en las políticas públicas (Ballester, 2011), es un concepto aún por delimitar y operacionalizar. El disenso terminológico, que implica un debate conceptual, se inicia con la reconciliación (Hochschild, 1989), presentada como la estrategia para solventar la doble jornada en el Programa Comunitario de Acción Social de 1974 (Ballester, 2011: 20). Transformada en conciliación en España, debido a su carga "económico empresarial" ha sido sustituida por corresponsabilidad en algunas esferas. Al margen de matices, en general, las de conciliación, "cobijan políticas públicas y privadas y tendencias de opinión diversas entre sí que conforman un debate de interés en Europa /.../ En este debate se superponen perspectivas temáticas distintas: la primera, la igualdad entre hombres y mujeres /.../ La segunda, la Estrategia Europea de empleo, y muy en particular, el incremento de la tasa de empleo femenina. La tercera, el reto demográfico que plantea el envejecimiento de la sociedad" (Cabeza, 2010: 46).

De hecho, según se enfoque uno u otra de estas perspectiva, en la relación familia-empleo, se tendrá en "una concepción distinta" (Fernández y Tobío, 2005: 53). Hay países centrados en facilitar la participación de la mujer en el mercado de trabajo que crean infraestructura y corresponsabilizan al estado de estos cuidados (Suecia, Finlandia, Dinamarca y Francia). Otros, consideran que son los ciudadanos (generalmente las mujeres) los responsables de la atención a la familia. Así, incentivan los tiempos parciales o las licencias remuneradas (Alemania o Austria) o sin remunerar (Holanda). (Chinchilla y León, 2011: 53). Otros tienen como fin la subida de la natalidad para asegurar al menos la tasa de reposición, es el caso de Francia cuyas políticas natalistas van dirigidas a las familias. De modo que, según enfoquemos el problema de la conciliación obtendremos unos resultados u otros. Este estudio tiene como objetivo los encuadres jurídicos, mediáticos y políticos del problema de la conciliación en España. El fin es sacar a la luz cómo se ha construido el problema de la conciliación vida familiar y laboral en la década que va de 1999 a 2009.

\section{Metodología}

Con el objeto de cumplir nuestras metas, diseñamos varios estudios, que se sirven de métodos diferentes:

1. En primer lugar, se plantea realizar un análisis textual de las principales leyes que afectan a la relación familia y trabajo en España: Ley 39/1999 de 5 de noviembre de Conciliación Vida Familiar y Laboral y Ley 3/2007 de 22 de marzo para la Igualdad Efectiva de Mujeres y Hombres. 
2. En segundo lugar, haremos un análisis de las informaciones publicadas en los tres diarios españoles de mayor tirada (El País, El Mundo y ABC), también en un período de tiempo que va desde 1999 a 2009.

3. En tercer lugar, proponemos acometer un análisis de contenido de la agenda política, en concreto las iniciativas parlamentarias que ha habido sobre conciliación durante doce años, desde 1996 a 2008², a través de las actas del Congreso y del Senado

\section{Fuentes}

Para el primer estudio se ha analizado el contenido de la Ley 39/1999, de 5 de noviembre, de Conciliación de la Vida Familiar y Laboral de las Personas Trabajadoras. BOE ${ }^{\circ} 266$, de 6 de noviembre de 1999 y la Ley 3/2007, de 22 de marzo, para la Igualdad Efectiva de Mujeres y Hombres. BOE ${ }^{\circ}{ }^{71}$, de 23 de marzo de 2007.

Para el segundo estudio, y con respecto a la agenda mediática, nos hemos servido de las noticias disponibles en las hemerotecas on-line de los diarios El Mundo, el $A B C$ y el País (los tres periódicos de mayor difusión media en el ámbito nacional según la OJD) desde 1996 a 2008. El criterio de selección de noticias ha sido el de la aparición de alguna o varias de las siguientes palabras clave en el título o subtítulo de la noticia: "conciliación familia-trabajo", "conflicto familia-trabajo", "igualdad", "balance laboral" y "balance familiar". El corpus de textos seleccionados está compuesto por todas las noticias publicadas en cualquier sección de las ediciones regulares de los diarios escogidos, incluidas las ediciones provinciales y excluidos los suplementos y los dominicales. Se han analizado un total de 1232 noticias: 352 de $A B C, 205$ de $E l$ Mundo, 675 de El País.

Para el tercer estudio, la fuente de información ha sido el Diario de Sesiones del Congreso de Diputados ${ }^{3}$, en el que se ha realizado una búsqueda de las palabras "conciliación" y "conflicto familiar-laboral" para recoger todas las iniciativas legislativas, intervenciones, propuestas de ley orgánica, propuestas no de ley, informes, ponencias, enmiendas, preguntas orales y escritas al gobierno sobre conciliación vida familiar y laboral. Han sido analizadas todas las actas de las sesiones desarrolladas desde marzo de 1996 hasta marzo de 2008, arco de tiempo que en términos legislativos se corresponde con la VI legislatura (años 1996-2000); VII legislatura (años 2000-2004) y VIII legislatura (años 2004-2008). Se han reunido un total de 97 actas parlamentarias en las que el tema objeto de estudio, conciliación, aparece mencionado en el orden del día ${ }^{4}$.

\section{Desarrollo}

2 Teniendo en cuenta que 1995 es el año de la IV Conferencia de Pekín y punto de partida de las estrategias de mainstreaming de género y que un año después, 1996, es cuando la UE promulga la Directiva sobre el permiso parental.

3 Diario de Sesiones del Congreso de Diputados http://www.congreso.es

${ }^{4}$ Las Cortes Generales están constituidas por dos cámaras principales: el Congreso y el Senado. Éstas, a su vez, se componen de otros órganos, las llamadas Comisiones, de mayor especialización en los asuntos a tratar. Hasta finales del siglo XIX el único órgano de las Cortes era el Pleno, a partir del XX comenzaron a desarrollarse las Comisiones y éstas se definen como órganos preparatorios que tienen atribuidas para determinados casos facultades decisorias (Martínez, 2000: 85) pueden ser Permanentes y no Permanentes, Legislativas y no Legislativas. 


\subsection{Respecto al análisis jurídico}

Propiamente, los antecedentes jurídicos para la regulación de la conciliación están en la Constitución de 1978, que reconoce la capacidad laboral en términos de igualdad, la no discriminación y la protección de la familia en sus artículos 14, 39.1, 35 y 9.2. Más recientemente, aparecen la Ley 3/1989 de 3 de marzo sobre maternidad e igualdad de trato de la mujer trabajadora y la Ley 31/1995 de prevención de riesgos laborales que introduce, en su artículo 26, previsiones especiales para la situación de embarazo y lactancia. Pero será la Ley 39/1999 de Conciliación de la Vida Familiar y Laboral el punto de referencia jurídico del problema de la conciliación en España (Guirao, 2010: 132).

\subsubsection{La Ley 39/1999 de Conciliación Vida familiar y Laboral}

La Ley 39/1999, de 5 de noviembre, para promover la conciliación de la vida familiar y laboral de las personas trabajadoras (LCVFL) tiene como objetivos: acoger preceptos comunitarios pendientes de transposición; dar cumplimiento al mandato constitucional (Art. 14, 9.2 y 39.1 de la Constitución); guardar equilibrio para que los permisos por maternidad y paternidad no afecten al empleo, reducir la jornada para el cuidado de familiares; y facilitar que los trabajadores puedan trabajar y al mismo tiempo satisfacer sus obligaciones familiares. La norma introduce novedades con respecto al ordenamiento jurídico que se resumen a continuación. Las principales novedades que introduce la norma con respecto al ordenamiento jurídico anterior tienen que ver con a) la prestación/suspensión por maternidad ${ }^{5}$ b) la incapacidad temporal por riesgo durante el embarazo ${ }^{6}, \mathrm{c}$ ) las protecciones frente al despido, d) las excedencias por cuidado de hijos y familiares ${ }^{7} \mathrm{y}$ e) los permisos parentales ${ }^{8}$.

5 La LCVFL ha introducido, respecto a la suspensión por maternidad algunos cambios esenciales:

- Con relación al parto múltiple, la LCVFL amplía la duración de la suspensión, 16 semanas, en dos semanas más por cada hijo a partir del segundo. La misma aplicación se produce en el caso de adopciones o acogimientos múltiples.

- Con relación a la baja por maternidad, la madre puede transferir al padre parte de la suspensión hasta diez semanas ininterrumpidas, que pueden distribuirse entre padre y madre con casi total flexibilidad (disfrute simultáneo o sucesivo; disfrute a tiempo completo o a tiempo parcial).

- En caso de adopción o acogimiento, la LCVFL prolonga la suspensión por maternidad hasta las 16 semanas en hijos menores de 6 años y contempla el caso de extender la suspensión a hijos mayores discapacitados o con problemas de integración social acreditados por los servicios sociales.

- Con relación al disfrute de la suspensión por maternidad, por parte del padre, cuando la madre fallece. la LCVFL contempla el derecho del padre a disfrutar de todo el permiso de maternidad que no hubiese podido disfrutar la madre fallecida.

6 El articulo 10 de la Directiva Comunitaria 92/85 fija las principales situaciones que ha de contemplar la protección frente al despido de la mujer embarazada, que ha dado a luz o en periodo de lactancia.

- Despido que recae sobre trabajadores durante la suspensión por maternidad, adopción, acogimiento o riesgo durante el embarazo. 


\subsubsection{Ley 3/2007 para la Igualdad Efectiva de Mujeres y Hombres}

La Ley Orgánica 3/2007, de 22 de marzo, para la Igualdad Efectiva de Mujeres y Hombres (LOIEMH) sitúa a España, al menos simbólicamente, entre los países de vanguardia en políticas de igualdad de género. La Ley, que tiene como objetivo la prevención y eliminación de discriminaciones por razón de sexo y la previsión de políticas activas para hacer efectivo el principio de igualdad, procede de la transposición de tres directivas: 2002/73/CE, 2004/113/CE, y 97/80/CE del Consejo. Integrada por 78 artículos, anhela ser una "Ley código". En sus disposiciones modifica el núcleo duro del Derecho del Trabajo y de la Seguridad Social: el Estatuto de los Trabajadores, la Ley General de la Seguridad Social, la Ley de Procedimiento Laboral, la Ley de Prevención de Riesgos Laborales.

La LOIEMH fomenta la corresponsabilidad en la asunción de obligaciones familiares, reconoce explícitamente la conciliación como un derecho de los trabajadores en el mundo productivo (Art. 44) y sienta por vez primera las bases del permiso de paternidad. Asimismo, establece la obligación de integrar entidades y documentos para evitar la discriminación laboral entre mujeres y hombres (incluidas también las derivadas de los conflictos familiares-laborales), en "un plan de igualdad /.../ objeto de negociación" (Art. 45.2). Este "conjunto ordenado de medidas, adoptadas después de realizar un diagnóstico de situación, tendentes a alcanzar en la empresa la igualdad de trato y de oportunidades entre mujeres y hombres y a eliminar la discriminación por razón de sexo" (Art. 46.1) debe fijar los objetivos a alcanzar y las estrategias y prácticas para lograrlos y sistemas de seguimiento y evaluación.

\subsection{Respecto al análisis mediático}

- Despido de trabajadoras embarazadas, desde el inicio del embarazo hasta el comienzo del periodo de suspensión correspondiente.

- Despido que recaiga sobre trabajadores que hubieran solicitado o se encuentran disfrutando del permiso de lactancia, la reducción de jornada por guarda legal o cuidado de enfermos, así como la excedencia por cuidado de hijos o familiares.

7 Son tres, fundamentalmente, las innovaciones que ha supuesto la ley 39/1999 en la regulación de la excedencia familiar (Ballester, 2000: 58 ss): 1) la creación de la figura de excedencia por cuidado de familiares tiene el mismo régimen jurídico que la figura de cuidado de hijos menores; 2) la excedencia como derecho individual y su posible disfrute simultáneo; 3 ) el inicio del cómputo de la excedencia de adopción o acogimiento se equipara al de maternidad.

8 En este punto, la LCVFL introduce tres nuevos permisos parentales: 1) Permiso de lactancia: es un permiso retribuido a cargo del empresario, reconocido a la trabajadora con un hijo menor de nueve meses, puede pasar al padre en caso de que ambos trabajen. 2) Reducción de jornada por guarda legal. 3) Permiso por fuerza mayor familiar. De igual manera, las modificaciones introducidas por permisos de fuerza mayor familiar se refieren sólo a las causas que originan estos premisos, que finalmente quedan en: accidente, enfermedad grave u hospitalización de parientes hasta el segundo grado de consanguinidad, en cuyo caso existe un permiso retribuido de dos días. 
Respecto a la cobertura periodística, en la construcción del problema de la conciliación vida familiar y laboral en España, se pueden distinguir dos etapas. Una primera hasta marzo de 2004, con un ciclo de noticiabilidad bajo y en el que la que la cobertura periodística se centra en definir el problema de la conciliación y situarlo en el espacio público. Y una segunda etapa, a partir de 2004 y hasta el 2007, en el que sube el ciclo de noticiabilidad, vinculado a la aparición en prensa de noticias relacionadas con el tema de la igualdad. Es a partir del $2007^{9}$, cuando los medios aparecen plenamente concienciados del problema, con el mayor índice de publicación de noticias, y plenamente reconocidos como instrumentos útiles en la construcción del problema de la conciliación. Por tanto, la visibilidad del tema en la agenda fue aumentado poco a poco en estos años. En general, no se dio la omnipresencia del problema en el conjunto de los diarios. Como se deduce en la inestabilidad de la suma de menciones a lo largo de los meses, la acumulación en el tiempo de las informaciones no ha sido progresiva y existen diferencias en la atención global prestada al problema de la conciliación. "Estas desemejanzas son importantes porque, como se ha visto, la acumulación de un tema en la agenda de un medio es reflejo de la importancia que éste le ha conferido, y puede condicionar la importancia relativa de esa cuestión para sus audiencias" (Martín Llaguno, 2002: 135).De hecho, la atención prestada a la conciliación difirió entre cabeceras: el diario que más noticias publicó fue El País, seguido del Mundo y de $A B C$. La acumulación de noticias se produjo de manera paralela en El Mundo y el $A B C$, que experimentaron un ligero aumento en las informaciones de la VI legislatura a la VII legislatura, y que se pronunció entre la VII y la VIII legislatura. Por su parte el País siguió una tendencia diferente, acumulando textos sobre el tema a más velocidad que los otros medios especialmente en la VIII legislatura.

Grafico ${ }^{\circ}{ }^{1}$ : Ciclos de noticiabilidad: Presencia del tema en los diarios, 1996-2008

Fuente: El País, el Mundo, ABC, años 1996-2008. Elaboración propia a partir del pro-

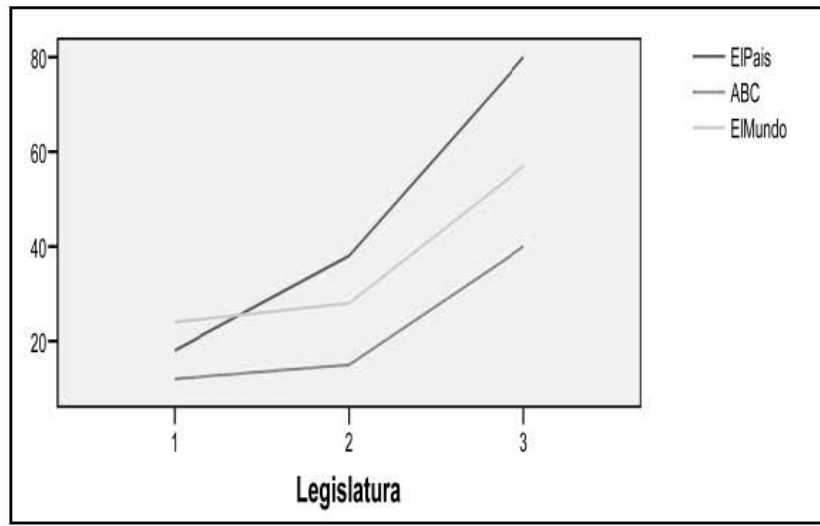

yecto La construcción social del problema del conflicto trabajo-familia. Análisis mediático, político y de la realidad social. Plan Nacional I+D+I.

En cuanto al contenido y los enfoques con los que se construyó el problema de la

${ }^{9}$ Ciclo que coincide con la aparición de la Ley de Igualdad y el segundo gobierno de Zapatero. 
conciliación en prensa durante estos doce años, hemos de volver a mencionar primero, el hecho de que la mayor parte de las informaciones problematizaron a las mujeres en exclusiva. Un 53,2 \% enfocó la noticia como un problema de asignación de roles a géneros, un $35,6 \%$ de las noticias analizadas no enfocó este tema. Un 30,4\% de las noticias analizadas problematizaron al trabajo como causa del conflicto y un $11,5 \%$ problematizaron a la familia. En cambio, y siguiendo el análisis de encuadres que durante los años 1996 a 2008, hemos realizado a los periódicos de mayor tirada (ABC, el Mundo y El País), un 58\% de las noticias analizadas ubicaban el problema de la conciliación en ambos espacios: la familia y el trabajo, mientras que un 59,5\% de las noticias ubicaba el problema de la conciliación en el ámbito laboral y un 40,5\% lo hacía en el familiar (El País, el Mundo, ABC, 1996-2008).

Por otro lado, un alto porcentaje de las noticias analizadas sitúan a las mujeres como receptoras del problema, 40,9\%. Las mujeres son tratadas como las principales responsables del cuidado y ellas son las que tienen el problema de conciliar vida laboral y familiar. Eso explica que sólo un $15,1 \%$ de noticias analizadas atribuya a los hombres el problema de la conciliación. (El País, el Mundo, ABC, 1996-2008).

\subsection{Respecto al análisis político}

La visibilidad del tema en la agenda política ha aumentado en estos años. En cuanto a la presencia relativa del tema por años, en la primera década se observa una tendencia creciente que alcanza su máximo en 2006 -con una tasa de 1.02 por 1000-, que empieza a caer en picado en 2007, cerrando un ciclo de interés parlamentario sobre el tema. Por legislatura, la eclosión del tema se da en la VIII, donde el tema alcanza la tasa máxima de 0,68 iniciativas sobre el tema por cada 1000 .

La VIII legislatura (años 2004-2008) viene marcada por el ascenso del partido socialista al poder, y por el desarrollo de las políticas de igualdad de género, lo que produce que en la agenda política el tema de igualdad entierre políticamente al de la conciliación. Durante esta legislatura se producen una serie de cambios normativos importantes: aprobación de la Ley Integral contra la Violencia de Género, de 28 de diciembre de 2004. Creación del Consejo Estatal y del Observatorio Estatal de Familias, de 11 de mayo de 2007.

Gráfico $\mathrm{n}^{\circ} 2$ : Por año, tasa de iniciativas sobre conciliación $(1997-2007)$

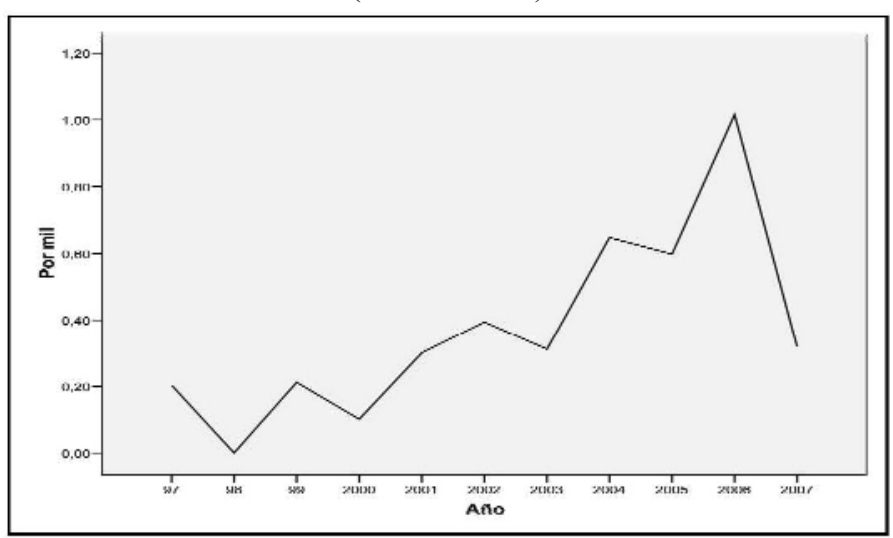

Fuente: Elaboración propia a partir del proyecto: La construcción social del problema del conflicto trabajo-familia. Análisis mediático, político y de la realidad social. Plan Nacional $I+D+I$. 
Ley por la que se modifica el Código Civil en materia de Separación y Divorcio, de 26 de noviembre de 2004, que da posibilidad de acceso directo al divorcio sin separación previa. Ley por la que se modifica el Código Civil para permitir el matrimonio de personas del mismo sexo, de 2 de julio de 2007. Aprobación de la Ley para la Igualdad Efectiva de Mujeres y Hombres, marzo de 2007, que introduce reformas en materia de conciliación. Finalmente, la puesta en marcha del "Plan Concilia", en 2006, que afecta a más de 500.000 empleados públicos de la Administración del Estado e incluye mejoras en los regímenes de permisos y horarios para conciliar la vida familiar y la laboral. (Martín Llaguno y Guirao, 2012)

Dado que muchas de las iniciativas fueron preguntas escritas al gobierno $51,7 \%$, no se tramitaron en sesiones parlamentarias. De las que sí se tramitaron, el 13,4\% pasó por Pleno y el 33,6\% en Comisión. Entre las iniciativas debatidas en Comisión, la mayoría se discutieron en la de Trabajo y Asuntos Sociales $(n=40), 6$ en la Comisión Mixta de Derechos de la Mujer, 2 en la Comisión de Política Social y Empleo, 1 en la Comisión de Justicia y 1 en la de Defensa. (Congreso de los Diputados. Años 1996 a 2008).

Así, el 35\% de las iniciativas parlamentarias sobre conciliación fueron promovidas por las diputadas y senadoras, frente a un $22 \%$ que fueron realizadas por diputados y senadores y un $42 \%$ en las que no se pudo identificar el sexo del autor. (Congreso de los Diputados. Años 1996 a 2008).

El grupo parlamentario Popular presentó la mayoría de iniciativas (40\%), seguido de la Comisión de Trabajo y Asuntos Sociales (22\%) y el grupo Socialista (19,5\%). El grupo de Izquierda Unida aparece en cuarto lugar $(7,4 \%)$, seguido de Ezquerra Republicana $(2,7 \%)$ y el grupo Catalán $(2 \%)$. De hecho, la conciliación ha sido un tema más vinculado al grupo Popular que al Socialista. (Congreso de los Diputados. Años 1996 a 2008).

\section{Conclusiones}

En definitiva, el marco jurídico construido, cambiante dependiendo del partido en el gobierno, ha mantenido las constantes del encuadre del tema en las políticas de género, y en la persecución del pleno empleo. Así, las acciones - centradas casi en exclusiva en provisiones en el trabajo o en medidas incentivación de la natalidad- se han dirigido a la mujer y han mantenido estereotipos de género. A grandes rasgos, y como conclusión de este análisis jurídico, podemos distinguir dos modelos de políticas familiares en España. Políticas tendentes a la conciliación, que incluyen medidas dirigidas fundamentalmente a las mujeres (prestaciones sociales, permisos, excedencias y ayudas fiscales). Estas políticas institucionalizan el hecho de que son las mujeres las que han de asumir las tareas de conciliación, no cuestionando la asignación de roles a géneros. Este es el caso de la Ley de Conciliación 33/1999. El segundo modelo de políticas familiares son políticas tendentes a la igualdad (Ley para la Igualdad Efectiva de Mujeres y Hombres, 2007), que implementan medidas para desvincular a la mujer del espacio doméstico y ubicarla en el espacio público (permiso de paternidad, acciones positivas, paridad en los órganos de representación, permisos parentales remunerados, servicios públicos de atención y cuidado) con un modelo de sustenta- 
dor/cuidador igualitario, y que implican al hombre en las tareas de corresponsabilidad. (Guirao, 2011: 23).

Respecto al análisis mediático y político, señalar que en primer lugar, la mayor parte de las informaciones problematizaron a las mujeres en exclusiva $(40,9 \%)$, o junto con los hombres $(44,1 \%)$, y sólo un $15 \%$ de las noticias incidió en que la conciliación era un problema masculino. Segundo, en la construcción mediática del problema de la conciliación vida familiar y laboral en España, el concepto conciliación ha cambiado su significado. Este concepto que se introdujo al principio en los medios como un problema de la mujer, relacionado con el libre mercado y con el mundo laboral, en España, al contrario que en Europa, ha cambiado su sentido y su significado, y aparece progresivamente vinculado al tema de la igualdad de género.

Finalmente, en la historia política del problema de la conciliación vida familiar y laboral destaca la lentitud de las iniciativas parlamentarias, sobre todo en la VI (años 1996 a 2000) y VII (2000 a 2004) legislaturas que se corresponden con el primer gobierno y el segundo del Partido Popular. En la VII legislatura (2004-2008), aumentan las iniciativas parlamentarias y se aceleran las medidas a tomar sobre políticas familiares, siendo éste uno de los temas estrella del gobierno del Partido Socialista, y en políticas familiares se cambia la perspectiva de género que pasa ahora de la conciliación a la igualdad, lo que denota un punto de vista nuevo del problema. Así, hemos visto que el $40 \%$ de las iniciativas presentadas sobre conciliación durante estos 12 años, fueron presentadas por el Partido Popular en el gobierno durante la VI y la VII legislatura, frente a un 19,5\% del Partido Socialista y un $22 \%$ de la Comisión de trabajo y Asuntos Sociales. Esto nos indica que la conciliación en la agenda política ha sido más un tema propio del Partido Popular que del Partido Socialista. De hecho, cuando hablamos de cambio cualitativo en la construcción política del problema de la conciliación nos referimos al cambio en la percepción del esquema tradicional de adjudicación de roles en función de género que subyace a muchas de las iniciativa parlamentarias y que está presente en la Ley de Conciliación. Este cambio cualitativo se observa primero en el hecho de que durante el arco de tiempo analizado, se ha producido sólo una incipiente construcción política del problema de la conciliación, decayendo en el 2008 sin llegar a fraguar en verdaderas estrategias. Y, segundo, en la sustitución de "la conciliación" como concepto fundamental que guía las políticas públicas familiares por "la igualdad". Así, con el ascenso del Partido Socialista al poder en marzo de 2004, VIII legislatura, desaparece de la construcción política el primero de los términos que queda sustituido por el segundo. Este cambio parece implicar también el impulso del desarrollo de medidas dirigidas no sólo a las mujeres, sino también a los hombres, a partir de su mayor implicación en la corresponsabilidad familiar y doméstica.

\section{Referencias bibliográficas}

BALLESTER, María Amparo (2000): La Ley 39/1999 de Conciliación de la Vida Familiar y Laboral: una corrección de errores con diez años de retraso. Valencia, Tirant lo Blanc. 
BALLESTER, María Amparo (2011): “Conciliación y corresponsabilidad en la Unión Europea" en CABEZA, Jaime (ed.): Conciliación de la vida familiar y laboral y corresponsabilidad entre sexos. Valencia, Tirant lo Blanc, pp. 17-49.

CABEZA, Jaime (2010): “Conciliación de la vida privada y laboral". Temas laborales 103, pp. 45-65.

CHINCHILLA, Nuria y LEÓN, Consuelo (2011): Diez años de conciliación en España. Madrid, Grupo 5.

FERNÁNDEZ, Juan Antonio y TOBÍO, Constanza (2005): Conciliar las responsabilidades familiares y laborales: politicas y prácticas sociales. Documentos de trabajo 79/2005. Madrid, Fundación Alternativa.

GUIRAO, Cristina (2010): La construcción social del problema de la conciliación vida familiar y laboral en España, 1999-2009. Tesis Doctoral. Dirección: Marta Martín Llaguno. Alicante, Universidad de Alicante.

GUIRAO, Cristina (2011): "Políticas de conciliación y políticas de igualdad. El caso español". Aposta, revista de ciencias sociales. $\mathrm{N}^{\circ}$ 49, pp. 1-37.

HOCHSCHILD, Arlie Russell (1989): The Second Shift: Working Parents and the Revolution at Home. New York, Viking

MARTÍN LLAGUNO, Marta (2002): La función de recuerdo de los medios de difusión: estudio de las condiciones de posibilidad del efecto. El caso del SIDA en la prensa. Alicante. Universidad de Alicante.

MARTÍN LLAGUNO, Marta y GUIRAO MIRÓN, Cristina (2012): "El debate parlamentario sobre conciliación laboral y familiar en España: análisis de una década de iniciativas". Revista Alternativas. Cuadernos de trabajo social. Alicante. Universidad de Alicante.

MARTíNEZ, Antonia (2000): El Congreso de los Diputados en España. Madrid. Tecnos.

\section{Legislación}

Ley 39/1999, de 5 de noviembre, para promover la conciliación de la vida familiar y laboral de las personas trabajadoras, BOE 266, de 6 de noviembre de 1999, pp. 38934-38942.

Ley 39/2006, de 14 de diciembre, de promoción de la autonomía personal y atención a las personas en situación de dependencia, BOE 299, de 15 de diciembre de 2006, pp. 44142-44156.

Ley $9 / 2009$, de 6 de octubre, de ampliación de la duración del permiso de paternidad en los casos de nacimiento, adopción o acogida, BOE 242, de 7 de octubre de 2009, pp. 84692-84694.

Ley Orgánica 3/2007, de 22 de marzo, para la igualdad efectiva de mujeres y hombres. BOE 71, de 23 de marzo de 2007, pp. 12611-12645. 


\section{Cristina GUIRAO MIRÓN}

Catedrática de EESS

Profesora asociada Dep. Sociología y Política Social

Universidad de Murcia

Facultad de Economía y Empresa

30100 Espinardo

Tel. 968887829

cguirao@um.es

\section{Marta MARTÍN LLAGUNO}

Catedrática de Universidad Comunicación Audiovisual y Publicidad Universidad de Alicante

Departamento de Comunicación y Psicología Social

Edificio de CC. Sociales

Campus Universitario, San Vicente del Raspeig, (Alicante)

Tél: 965903869

marta.martin@ua.es 